\title{
Efficacy of Transcartilaginous Electrodes for Intraoperative Neural Monitoring During Thyroid Surgery
}

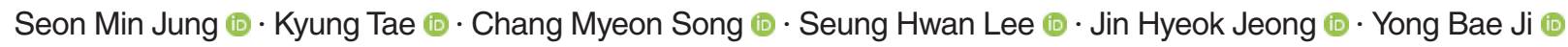 \\ Department of Otolaryngology-Head and Neck Surgery, Hanyang University College of Medicine, Seoul, Korea
}

Objectives. This study was conducted evaluate the efficacy of electromyography (EMG) using transcartilaginous (TC) electrodes through the thyroid cartilage and perichondrium.

Methods. We prospectively collected EMG data from intraoperative neuromonitoring (IONM) of 54 nerves at risk in 38 patients during thyroidectomy. We followed standardized IONM procedures in all operations. EMG signals from both endotracheal tube (ET) electrodes and TC needle electrodes were recorded simultaneously. We compared the characteristics of the EMG signals and the efficacy of both methods.

Results. Significantly higher mean EMG amplitudes were recorded by TC electrodes than by ET electrodes in all four-step procedures (V1-R1-R2-V2, $P<0.001)$. Loss of signal (LOS) occurred in five patients in ET electrodes, but in only two patients in TC electrodes. Postoperative laryngoscopy revealed recurrent laryngeal nerve palsy in the two patients who showed LOS from both the ET and TC electrodes, and vocal cord movement was intact in the other three patients. Therefore, the positive predictive values of LOS in ET and TC electrodes were $40 \%$ and $100 \%$, respectively.

Conclusion. EMG recording using TC needle electrodes is feasible and effective, making it a good alternative technique for IONM.

Keywords. Intraoperative Neurophysiological Monitoring; Thyroidectomy; Recurrent Laryngeal Nerve; Electrode

\section{INTRODUCTION}

Recurrent laryngeal nerve (RLN) injury is one of the most important and serious complications of thyroid surgery, with a reported incidence of $0 \%-11 \%$ [1]. Visual identification of the RLN is the gold standard for finding the RLN and avoiding RLN palsy $[2,3]$. However, even expert thyroid surgeons cannot be entirely free from this complication. Therefore, various types of intraoperative neuromonitoring (IONM) have been developed to facilitate the identification of the RLN and to verify its func-

\footnotetext{
- Received September 16, 2019

Revised December 18, 2019

Accepted January 7, 2020

- Corresponding author: Yong Bae Ji

Department of Otolaryngology-Head and Neck Surgery, Hanyang

University College of Medicine, 222-1 Wangsimni-ro, Seongdong-gu,

Seoul 04763, Korea

Tel: +82-2-2290-8580, Fax: +82-2-2293-3335

E-mail: jyb20000@hanyang.ac.kr
}

tional integrity [4-6].

Electrical stimulation to identify the RLN during thyroidectomy was introduced in the late 1960s and has become widely used $[7,8]$. In the early period, surgeons proposed various IONM methods. Some surgeons palpated the movement of the larynx (especially the posterior hypopharyngeal wall) during thyroidectomy, and some recorded the pressure of an endotracheal tube (ET) cuff that was placed between the vocal cords. Attempts were also made to obtain EMG signals from the laryngeal muscle using needle electrodes or surface electrodes in different positions [9-12]. Initially, needle electrodes were used due to their stability. However, the electromyography (EMG) ET with a surface electrode was developed and released commercially in the 1990s $[11,13]$. EMG ETs have been widely used for thyroid and parathyroid surgery and have emerged as a standard method for IONM because they are easy to set up $[5,8,14]$. A standardized process for IONM that systematically identifies signals from the vagus nerve and the RLN in order (V1, R1, R2,V2) was published

Copyright $\odot 2020$ by Korean Society of Otorhinolaryngology-Head and Neck Surgery.

This is an open-access article distributed under the terms of the Creative Commons Attribution Non-Commercial License (https://creativecommons.org/licenses/by-nc/4.0)

which permits unrestricted non-commercial use, distribution, and reproduction in any medium, provided the original work is properly cited. 
by the International Intraoperative Monitoring Study Group in 2010 [15].

However, EMG ET electrodes have several limitations. First, they are basically surface-contact electrodes [16,17], which must be in contact with the mucosal surface of the vocal cord to obtain the proper evoked potential from the laryngeal muscles. The ET should be large enough to achieve proper contact between the electrodes and the vocal cords. However, this can sometimes be difficult due to the limited size of ETs. Furthermore, proper positioning of the EMG tube is very important for successful IONM.The optimal position of the EMG tube should be checked after the patient assumes the neck extension position for thyroidectomy. Displacement and rotation of the EMG tube during thyroidectomy can result in a low-voltage EMG signal, or even monitoring failure or a false loss of signal (LOS) $[14,18,19]$. Therefore, not all changes in EMG amplitude represent the actual function of the RLN. Thus, the ET position must be verified if the EMG amplitude changes significantly during the operation, even if the RLN is grossly intact $[14,20]$. Other limitations of ET electrodes include their high cost and the difficulty of applying them to patients who have undergone a tracheostomy.

To overcome these limitations, some surgeons have suggested a method in which needle electrodes are inserted directly into the thyroid cartilage $[17,20]$. However, research on the effectiveness of this method is lacking. Therefore, we performed this study to evaluate the efficacy of IONM with subdermal needle electrodes inserted through the thyroid cartilage.

\section{MATERIALS AND METHODS}

We prospectively collected EMG data from IONM of 54 nerves at risk (NARs) in 38 patients during thyroidectomy. All surgeries were performed by a single surgeon (YBJ) from July 2017 to December 2018. The indications of IONM were recurred thyroid cancer, preoperative vocal cord palsy, preoperative central lymph node metastasis, extracapsular invasion, and diffuse thyroid goiter were included. We decide the indications of IONM

\section{H I G H L I G H T S}

- We prospectively collected electromyography (EMG) data from intraoperative neuromonitoring during thyroid surgery.

- EMG signals from endotracheal tube (ET) and transcartilaginous (TC) electrodes were obtained simultaneously.

- The EMG amplitudes of the TC electrodes were significantly higher than those of the ET electrodes.

- The positive and negative predictive values of loss of signal by TC electrodes were $100 \%$.

- TC electrodes are more effective and stable than ET electrodes. according to the recommendation of Korean national medical insurance system.

Vocal cord movement was checked with flexible laryngofiberscopy before and after surgery in all patients. The standard IONM procedure suggested by the International Intraoperative Monitoring Study Group was followed in all surgeries [15].V1 was defined as an initial EMG signal from the vagus nerve before RLN dissection, and R1 was the initial signal from RLN which was identified at the tracheoesophageal groove. R2 and V2 signals were obtained after thyroidectomy was completed.

All surgeries were performed under general anesthesia with orotracheal intubation. A nondepolarizing muscle relaxant (rocuronium $0.5-0.6 \mathrm{mg} / \mathrm{kg}$ ) was used only once at the induction of anesthesia, and there was no additional use of muscle relaxants during surgery. Each patient was intubated with an EMGreinforced ET (Medtronic Xomed, Jacksonville, FL, USA). The size of the EMG tube was chosen to fit as tightly as possible against both sides of the vocal cords. The position of the ET was confirmed routinely after neck extension with a portable flexible laryngofiberscope before the tube fixation. After the placement of the ground electrode, we verified that the impedance value was less than $5 \mathrm{k} \Omega$. A monopolar stimulator probe was used for nerve stimulation.

During the surgery, after the pyramidal lobe was dissected, the thyroid cartilage was exposed. A 22-mm twisted pair of needle electrodes (Medtronic Xomed) was inserted through the perichondrium and thyroid cartilage at the mid-portion of both thyroid alar cartilage, and they were fixed with suture ties (Fig. 1). When the needles were difficult to insert due to calcification of thyroid cartilage, they were inserted into a soft portion in another part of the cartilage or a perichondrium.

Impedance $(<5.0 \mathrm{k} \Omega)$ and impedance imbalance $(<1.0 \mathrm{k} \Omega)$ were checked to ensure appropriate mucosal contact. Pulsatile neural stimulation was used at $4 \mathrm{~Hz}$, duration $100 \mathrm{~ms}$ at 1 to $2 \mathrm{~mA}$ during thyroidectomy with a standard monopolar stimulating probe (Medtronic Xomed). The intensity of stimulation was increased up to 3-5 mA if there was no response at 1-2 $\mathrm{mA}$, especially for the vagus nerve stimulations. The event threshold was preset at $100 \mu \mathrm{V}$.

In all cases, EMG signals from both ET and transcartilaginous (TC) electrodes were recorded simultaneously (Fig. 2) to compare two methods in the same patient. We compared the characteristic of EMG signal at each of the four steps of the standard procedure (V1, R1, R2,V2) and efficacy of both techniques. The appropriate event threshold was set to $100 \mu \mathrm{V}$. LOS was noted when the largest amplitude was less than $100 \mu \mathrm{V}$ [15].

\section{RESULTS}

The patients' characteristics and surgical details are summarized in Table 1. Among the 38 patients, there were 25 women and 13 

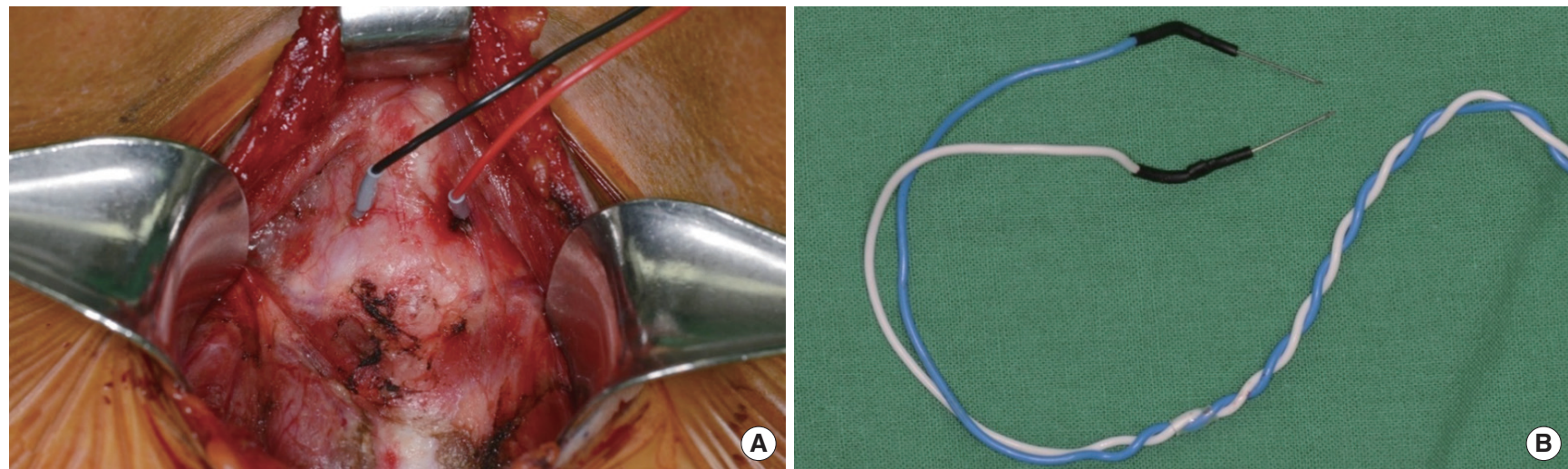

Fig. 1. Electrode placement. (A) Needle electrodes inserted through the thyroid cartilage. (B) A 22-mm twisted pair of needle electrodes (Medtronic Xomed, Jacksonville, FL, USA).

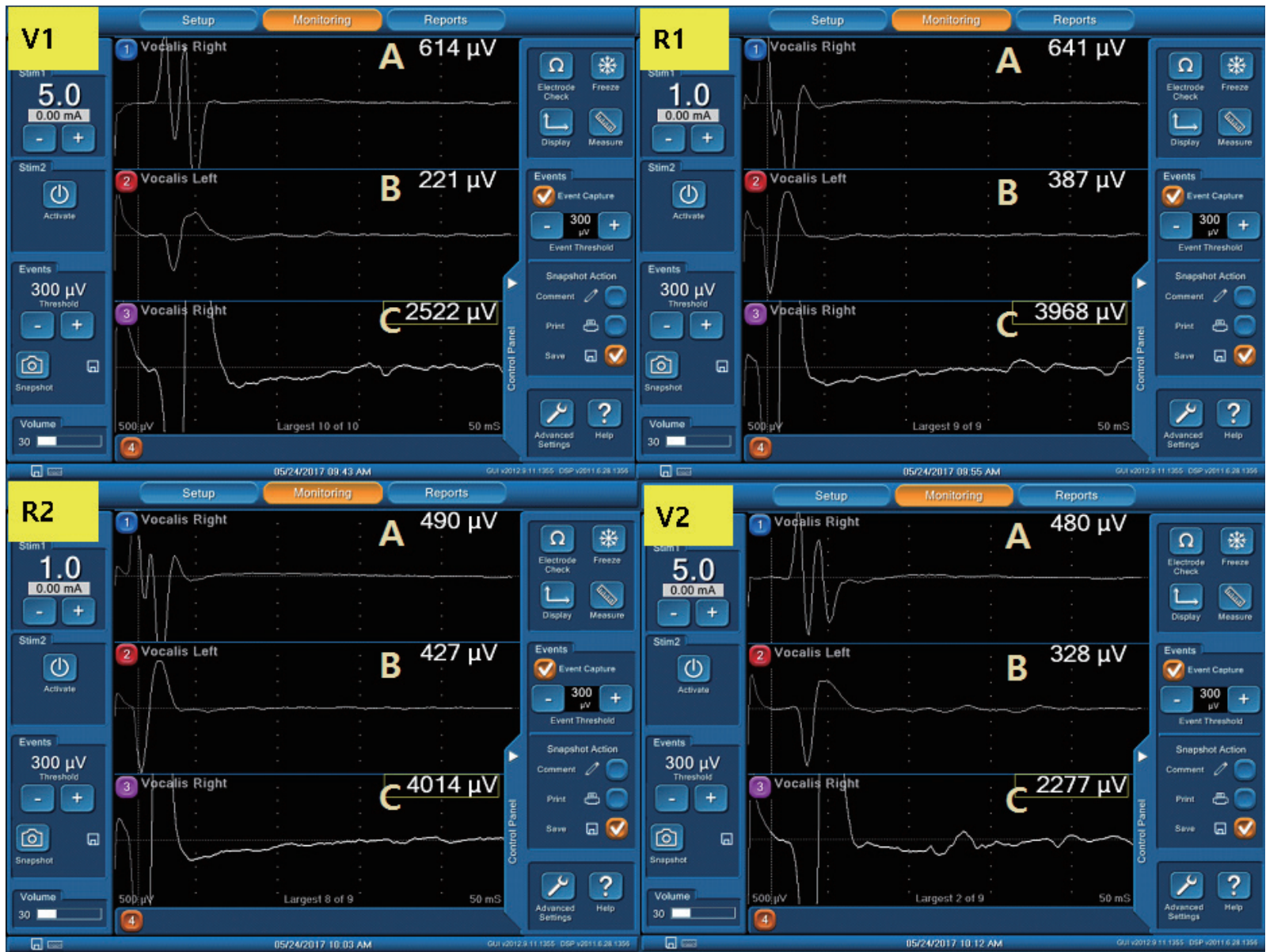

Fig. 2. Electromyography (EMG) signals from both the endotracheal tube and thyroid cartilage electrodes during each part of the standardized procedure (V1, R1, R2, V2). A, B: EMG signals from the right and left endotracheal tube electrodes, C: EMG signal from the thyroid cartilage electrodes.

men, with an average age of 53.8 years. In total, 21 patients underwent total thyroidectomy and 17 underwent thyroid lobectomy. The average time from intubation to $\mathrm{V} 1$ signal detection was $42.2 \pm 22.6$ minutes. AV1 amplitude $>500 \mu \mathrm{V}$ was achieved in 19 NARs (35.2\%) with ET electrodes and 47 NARs $(87.0 \%)$ with TC electrodes $(P<0.001)$. Significantly higher mean EMG amplitudes were recorded by TC electrodes than by ET electrodes in all four-step procedures (Table 2). 
Table 1. Clinicopathologic characteristics of the patients

\begin{tabular}{lc}
\hline Variable & Value $(\mathrm{n}=38)$ \\
\hline Sex & \\
Male & $13(34.21)$ \\
Female & $25(65.79)$ \\
Age $(\mathrm{yr})$ & $53.8 \pm 11.6$ \\
Pathology & \\
Papillary carcinoma & $20(52.63)$ \\
Follicular carcinoma & $1(2.63)$ \\
Medullary carcinoma & $1(2.63)$ \\
Benign & $16(42.11)$ \\
Type of surgery & \\
Total thyroidectomy & $21(55.26)$ \\
Thyroid lobectomy & $17(44.73)$ \\
Time interval between V1 and intubation (min) & $42.2 \pm 22.6$ \\
\hline
\end{tabular}

Values are presented as number (\%) or mean \pm standard deviation.

\section{Prediction of postoperative nerve function}

LOS occurred in five NARs $(9.26 \%)$ in the EMG signals measured with ET electrodes, but only two of them showed significant interval changes in the EMG signal measured with TC electrodes. The other three NARs exhibited no significant interval changes in the EMG signal measured with TC electrodes. Postoperative laryngoscopy revealed that the two NARs exhibiting LOS with both the ET and TC electrodes had RLN palsy. In contrast, the three NARs that displayed LOS only with the ET electrodes had intact vocal cord movement. Thus, the sensitivity, specificity, and positive and negative predictive values of LOS for the EMG signals from the ET electrodes were $100 \%$, 94\%, $40 \%$, and $100 \%$, respectively. However, these values were all $100 \%$ for the TC electrodes (Table 3 ).

Impending adverse EMG signals were found in two NARs that showed significant decreases in R2 amplitude ( $>50 \%$ from the R1 amplitude) with the ET electrodes. However, the amplitudes from the TC electrodes did not decrease in those cases, none of which showed vocal cord palsy on postoperative laryngeal examinations. There were no TC-electrode-related complications, such as bleeding, hematoma, or other injuries.

\section{DISCUSSION}

This TC neuromonitoring technique using a needle electrode was helpful for identifying the RLN, and we were able to obtain higher-amplitude EMG signals. Furthermore, this technique provided reliable IONM during thyroidectomy through a stable EMG amplitude regardless of the position of the patient or tube. Visual identification of the RLN alone is not sufficient to avoid RLN injury during thyroidectomy, because sometimes the RLN cannot be identified, and functional preservation of the RLN cannot be guaranteed even if the nerve seems grossly intact. For this reason, IONM during thyroid and parathyroid surgery was
Table 2. The amplitudes of electromyography signal from ET and TC electrodes

\begin{tabular}{lcrr}
\hline IONM & ET electrode $(\mu \mathrm{V})$ & TC electrode $(\mu \mathrm{V})$ & $P$-value \\
\hline V1 & $445.1 \pm 221.8(131-938)$ & $1,462.2 \pm 898.7(397-3,599)$ & $<0.001$ \\
R1 & $626.1 \pm 438.1(163-1,256)$ & $2,114.7 \pm 1,146.2(561-4,800)$ & $<0.001$ \\
R2 & $561.0 \pm 340.1(185-1,618)$ & $1,949.7 \pm 1,047.2(555-4,076)$ & $<0.001$ \\
V2 & $455.3 \pm 268.6(182-1,288)$ & $1,238.3 \pm 756.7(241-3,301)$ & $<0.001$ \\
\hline
\end{tabular}

Values are presented as mean \pm standard deviation (range).

ET, endotracheal tube; TC, transcartilaginous; IONM, Intraoperative neuromonitoring.

Table 3. Loss of signal for endotracheal and thyroid cartilage electrodes

\begin{tabular}{lccc}
\hline Variable & VC palsy (+) & VC palsy $(-)$ & Total \\
\hline ET electrode & & & \\
LOS (+) & 2 & 3 & 5 \\
LOS (-) & 0 & 49 & 49 \\
TC electrode & & & \\
LOS (+) & 2 & 0 & 2 \\
LOS (-) & 0 & 52 & 52 \\
Total & 2 & 52 & 54 \\
\hline
\end{tabular}

VC, vocal cord; ET, endotracheal tube; LOS, loss of signal; TC, transcartilaginous.

introduced $[8,21]$. When IONM was first used in thyroid surgery, several methods were employed, including needle electrodebased techniques [22-24]. Among these techniques, those involving commercially available ET electrodes became widely used.The International Neuromonitoring Study Group proposed standardized monitoring guidelines and recommended that surgeons follow a defined troubleshooting algorithm in the event of EMG signal problems during ET electrode-based surgery, because such problems may be a false signal from various causes, including ET problems [15].

However, whether IONM reduces the rate of postoperative vocal cord paralysis has remained controversial $[25,26]$. Some authors have reported that IONM is helpful for nerve identification and dissection [25,27-29]. A multivariable analysis of RLN palsy in 1,757 patients revealed that IONM protected against permanent RLN palsy, with an odds ratio [OR] of $0.43(95 \%$ confidence interval [CI], 0.19 to $0.93 ; P=0.033$ ), although it did not reach statistical significance for protecting against transient RLN palsy (OR, $0.67 ; 95 \% \mathrm{CI}, 0.44$ to $1.01 ; P=0.061$ ) [25]. However, other authors have reported that IONM did not reduce the vocal cord palsy rate, or that it was only effective in complicated cases, such as reoperations or those with a huge goiter [30,31]. Several meta-analyses have demonstrated that the use of IONM is associated with a reduced rate of transient RLN palsy, but not permanent RLN palsy [32-34].

This controversy may be due not only to the low incidence of RLN palsy, but also to the unreliable monitoring of RLN function. Unreliable monitoring causes surgeons to distrust EMG sig- 
nal changes and makes it difficult for them to determine a subsequent surgical strategy when LOS occurs. Monitoring may be unreliable for several reasons, but ET displacement is the most common reasons for a false adverse EMG signal because ET electrodes are contact electrodes. Several attempts have been made to overcome these limitations, such as by creating standardized guidelines or using different kinds of electrodes [21,35]. Recent reports showed that TC IONM using subdermal needle electrodes could be an effective alternative method $[17,20]$. Chiang et al. [20] prospectively evaluated an IONM technique in which paired needle electrodes were placed at the subperichondrium of the thyroid cartilage in 205 NARs. They reported that higher and more stable EMG amplitudes and fewer false EMG results were obtained through this technique [20]. An animal study using a porcine model also revealed that placing needle electrodes at the perichondrium of the thyroid cartilage enabled earlier identification of RLN injury and more stable monitoring [17].

We inserted the needle electrodes through the thyroid cartilage and obtained significantly higher EMG amplitudes from TC electrodes than from ET electrodes in all four-step procedures (V1-R1-R2-V2) $(P<0.001)$. Many surgeons and guidelines have stated that a sufficient initial V1 amplitude (at least $500 \mu \mathrm{V}$ ) is necessary for accurate monitoring [36,37]. In our study, V1 amplitudes $>500 \mu \mathrm{V}$ were obtained from 19 NARs (35.2\%) with ET electrodes and from 47 NARs $(87.0 \%)$ with TC electrodes; thus, a significantly higher proportion of TC electrode measurements met this criterion $(P<0.001)$. A high initial amplitude facilitates the early identification and localization of the RLN. During surgery, traction or electrical damage can cause the EMG amplitude to decrease, even when the nerve is grossly intact. Higher EMG amplitudes make it possible to monitor RLN function more sensitively. Thus, surgeons can obtain feedback from IONM, change their procedures, and be more careful when performing procedures near the RLN [37-39].

LOS is defined as a signal amplitude less than $100 \mu \mathrm{V}$ and can indicate that there has been severe damage to the RLN. However, several factors can influence the EMG signal, resulting in false positive or negative results. This can make decisions difficult, such as when LOS occurs on one side during a contralateral operation $[38,39]$. Therefore, it is very important to know whether the LOS is a true signal. In our study, the two NARs that exhibited LOS with both the ET and TC electrodes had postoperative RLN palsy. However, vocal cord movement was intact in the three NARs that displayed LOS with only the ET electrodes, so these were false-positive findings. As a result, the positive and negative predictive values were both $100 \%$ for the TC electrodes, while they were $40 \%$ and $100 \%$, respectively, for the ET electrodes.

The negative predictive value of IONM has reported to be been high in many studies, but the positive predictive value has varied from $12 \%$ to $88 \%$ [24,40-44]. This implies that various factors can cause false-positive LOS findings, but the most common cause is the inherent limitations of ET electrodes [33]. Our finding of a low positive predictive value for ET electrodes supports the notion that the position of the ET can be problematic. As TC electrodes enable stable monitoring without false positive or negative results, surgeons can use them confidently for IONM. Thus, TC electrodes can reduce unnecessary burdens during surgery and help surgeons to decide on a surgical strategy. In addition, TC electrodes have an economical benefit over ET electrodes. An ET with surface EMG electrodes costs about US \$300 in Korea, while TC electrodes only require an EMG needle, which costs less than $\$ 10$.

Despite these advantages, there are some limitations to TC electrodes. Firstly, TC electrodes are more invasive than ET electrodes $[17,20]$. Bleeding, hematoma, and other needle-related complications can occur. However, none of our patients experienced needle-related complications because the needles were thin. Secondly, needle electrodes can be difficult to insert if the patient's thyroid cartilage is calcified. However, in our study, the needle electrodes were inserted in all cases without much difficulty. Even if the needles cannot pass through the thyroid cartilage, the electrodes can be placed in the perichondrium of the thyroid cartilage, which can also provide enough EMG signal amplitude. In conclusion, TC electrodes are feasible and safe in IONM during thyroid surgery. This technique provides a more stable and reliable EMG signal than ET electrodes. Therefore, TC electrodes can be a good alternative to overcome the limitations of ET electrodes.

\section{CONFLICT OF INTEREST}

No potential conflict of interest relevant to this article was reported.

\section{ACKNOWLEDGMENTS}

This work was supported by the research fund of Hanyang University (HY-2013).

\section{ORCID}

Seon Min Jung

Kyung Tae

Chang Myeon Song

Seung Hwan Lee

Jin Hyeok Jeong

Yong Bae Ji https://orcid.org/0000-0003-1685-1574

https://orcid.org/0000-0002-0382-2072

https://orcid.org/0000-0001-5267-0135

https://orcid.org/0000-0003-2001-7689

https://orcid.org/0000-0001-8424-3998

https://orcid.org/0000-0002-0182-7865 


\section{AUTHOR CONTRIBUTIONS}

Conceptualization: YBJ. Data curation: SMJ, YBJ. Formal analysis: SMJ. Funding acquisition: YBJ. Methodology: YBJ. Project administration: YBJ. Visualization: YBJ. Writing-original draft: SMJ, YBJ.Writing-review \& editing: KT, CMS, SHL, JHJ.

\section{REFERENCES}

1. Vasileiadis I, Karatzas T, Charitoudis G, Karakostas E, Tseleni-Balafouta S, Kouraklis G. Association of intraoperative neuromonitoring with reduced recurrent laryngeal nerve injury in patients undergoing total thyroidectomy. JAMA Otolaryngol Head Neck Surg. 2016 Oct;142(10):994-1001.

2. Riddell VH. Injury to recurrent laryngeal nerves during thyroidectomy; a comparison between the results of identification and non-identification in 1022 nerves exposed to risk. Lancet. 1956 Sep;271(6944): 638-41.

3. Jatzko GR, Lisborg PH, Muller MG, Wette VM. Recurrent nerve palsy after thyroid operations--principal nerve identification and a literature review. Surgery. 1994 Feb;115(2):139-44.

4. Witt RL. Recurrent laryngeal nerve electrophysiologic monitoring in thyroid surgery: the standard of care? J Voice. 2005 Sep;19(3):497500 .

5. Chan WF, Lang BH, Lo CY. The role of intraoperative neuromonitoring of recurrent laryngeal nerve during thyroidectomy: a comparative study on 1000 nerves at risk. Surgery. 2006 Dec;140(6): 866-72.

6. Barczynski M, Konturek A, Cichon S. Randomized clinical trial of visualization versus neuromonitoring of recurrent laryngeal nerves during thyroidectomy. Br J Surg. 2009 Mar;96(3):240-6.

7. Flisberg K, LindholmT. Electrical stimulation of the human recurrent laryngeal nerve during thyroid operation. Acta Otolaryngol Suppl. 1969;263:63-7.

8. Gardner IH, Doherty GM, McAneny D. Intraoperative nerve monitoring during thyroid surgery. Curr Opin Endocrinol Diabetes Obes. 2016 Oct;23(5):394-9.

9. Rea JL. Postcricoid surface laryngeal electrode. Ear Nose Throat J. 1992 Jun;71(6):267-9.

10. Tschopp K, Probst R. New aspects in surgery of the thyroid gland with intraoperative monitoring of the recurrent laryngeal nerve. Laryngorhinootologie. 1994 Nov;73(11):568-72.

11. Mermelstein M, Nonweiler R, Rubinstein EH. Intraoperative identification of laryngeal nerves with laryngeal electromyography. Laryngoscope. 1996 Jun;106(6):752-6.

12. Khan A, Pearlman RC, Bianchi DA, Hauck KW. Experience with two types of electromyography monitoring electrodes during thyroid surgery. Am J Otolaryngol. 1997 Mar-Apr;18(2):99-102.

13. Maloney RW, Murcek BW, Steehler KW, Sibly D, Maloney RE. A new method for intraoperative recurrent laryngeal nerve monitoring. Ear Nose Throat J. 1994 Jan;73(1):30-3.

14. Kwak HY, Kim HY. The present and future of intraoperative neuromonitoring in thyroid surgery. Korean J Endocr Surg. 2014 Jun;14(2): 67-75.

15. Randolph GW, Dralle H; International Intraoperative Monitoring Study Group, Abdullah H, Barczynski M, Bellantone R, et al. Electrophysiologic recurrent laryngeal nerve monitoring during thyroid and parathyroid surgery: international standards guideline statement. Laryngoscope. 2011 Jan;121 Suppl 1:S1-16.

16. Tsai CJ,Tseng KY,Wang FY, Lu IC, Wang HM, Wu CW, et al. Electromyographic endotracheal tube placement during thyroid surgery in neuromonitoring of recurrent laryngeal nerve. Kaohsiung J Med Sci. 2011 Mar;27(3):96-101.

17. Zhao Y, Li C, Zhang D, Zhou L, Liu X, Li S, et al. Experimental study of needle recording electrodes placed on the thyroid cartilage for neuromonitoring during thyroid surgery. Br J Surg. 2019 Feb;106(3): 245-54.

18. Lu IC, Chu KS, Tsai CJ, Wu CW, Kuo WR, Chen HY, et al. Optimal depth of NIM EMG endotracheal tube for intraoperative neuromonitoring of the recurrent laryngeal nerve during thyroidectomy. World J Surg. 2008 Sep;32(9):1935-9.

19. Kim HY,Tufano RP, Randolph G, Barczynski M,Wu CW, Chiang FY, et al. Impact of positional changes in neural monitoring endotracheal tube on amplitude and latency of electromyographic response in monitored thyroid surgery: results from the porcine experiment. Head Neck. 2016 Apr;38 Suppl 1:E1004-8.

20. Chiang FY, Lu IC, Chang PY, Dionigi G, Randolph GW, Sun H, et al. Comparison of EMG signals recorded by surface electrodes on endotracheal tube and thyroid cartilage during monitored thyroidectomy. Kaohsiung J Med Sci. 2017 Oct;33(10):503-9.

21. Chiang FY, Lu IC, Chen HC, Chen HY,Tsai CJ, Hsiao PJ, et al. Anatomical variations of recurrent laryngeal nerve during thyroid surgery: how to identify and handle the variations with intraoperative neuromonitoring. Kaohsiung J Med Sci. 2010 Nov;26(11):575-83.

22. Spahn JG, Bizal J, Ferguson S, Lingeman RE. Identification of the motor laryngeal nerves: a new electrical stimulation technique. Laryngoscope. 1981 Nov;91(11):1865-8.

23. Brauckhoff M, Walls G, Brauckhoff K, Thanh PN, Thomusch O, Dralle $\mathrm{H}$. Identification of the non-recurrent inferior laryngeal nerve using intraoperative neurostimulation. Langenbecks Arch Surg. 2002 Jan;386(7):482-7.

24. Dralle H, Sekulla C, Lorenz K, Brauckhoff M, Machens A; German IONM Study Group. Intraoperative monitoring of the recurrent laryngeal nerve in thyroid surgery. World J Surg. 2008 Jul;32(7):135866.

25. Bergenfelz A, Salem AF, Jacobsson H, Nordenstrom E, Almquist M; Steering Committee for the Scandinavian Quality Register for Thyroid, Parathyroid and Adrenal Surgery (SQRTPA). Risk of recurrent laryngeal nerve palsy in patients undergoing thyroidectomy with and without intraoperative nerve monitoring. Br J Surg. 2016 Dec; 103(13):1828-38.

26. Pisanu A, Porceddu G, Podda M, Cois A, Uccheddu A. Systematic review with meta-analysis of studies comparing intraoperative neuromonitoring of recurrent laryngeal nerves versus visualization alone during thyroidectomy. J Surg Res. 2014 May;188(1):152-61.

27. Shedd DP, Burget GC. Identification of the recurrent laryngeal nerve. Arch Surg. 1966 Jun;92(6):861-4.

28. Chiang FY, Lu IC, Chen HC, Chen HY,Tsai CJ, Lee KW, et al. Intraoperative neuromonitoring for early localization and identification of recurrent laryngeal nerve during thyroid surgery. Kaohsiung J Med Sci. 2010 Dec;26(12):633-9.

29. Eltzschig HK, Posner M, Moore FD Jr. The use of readily available equipment in a simple method for intraoperative monitoring of recurrent laryngeal nerve function during thyroid surgery: initial experience with more than 300 cases. Arch Surg. 2002 Apr;137(4): 452-6.

30. Alesina PF, Rolfs T, Hommeltenberg S, Hinrichs J, Meier B, Mohmand $\mathrm{W}$, et al. Intraoperative neuromonitoring does not reduce the incidence of recurrent laryngeal nerve palsy in thyroid reoperations: results of a retrospective comparative analysis. World J Surg. 2012 Jun;36(6):1348-53.

31. Calo PG, Medas F, Gordini L, Podda F, Erdas E, Pisano G, et al. Interpretation of intraoperative recurrent laryngeal nerve monitoring signals: the importance of a correct standardization. Int J Surg. 2016 Apr;28 Suppl 1:S54-8. 
32. Rulli F, AmbrogiV, Dionigi G, Amirhassankhani S, MineoTC, Ottaviani $\mathrm{F}$, et al. Meta-analysis of recurrent laryngeal nerve injury in thyroid surgery with or without intraoperative nerve monitoring. Acta Otorhinolaryngol Ital. 2014 Aug;34(4):223-9.

33. Yang S, Zhou L, Lu Z, Ma B, Ji Q, Wang Y. Systematic review with meta-analysis of intraoperative neuromonitoring during thyroidectomy. Int J Surg. 2017 Mar;39:104-13.

34. Snyder SK, Hendricks JC. Intraoperative neurophysiology testing of the recurrent laryngeal nerve: plaudits and pitfalls. Surgery. 2005 Dec;138(6):1183-91.

35. Wu CW, Chiang FY, Randolph GW, Dionigi G, Kim HY, Lin YC, et al Transcutaneous recording during intraoperative neuromonitoring in thyroid surgery. Thyroid. 2018 Nov;28(11):1500-7.

36. Chavez KV, Ramirez J, Pantoja JP, Sierra M,Velazquez-Fernandez D, Herrera MF. Continuous intraoperative neural monitoring in thyroid surgery: a Mexican experience. Updates Surg. 2017 Dec;69(4):505-8.

37. Sarkis LM, Zaidi N, Norlen O, Delbridge LW, Sywak MS, Sidhu SB. Bilateral recurrent laryngeal nerve injury in a specialized thyroid surgery unit: would routine intraoperative neuromonitoring alter outcomes? ANZ J Surg. 2017 May;87(5):364-7.

38. Sitges-Serra A, Gallego-Otaegui L, Fontane J, Trillo L, Lorente-Poch L, Sancho J. Contralateral surgery in patients scheduled for total thyroidectomy with initial loss or absence of signal during neural monitoring. Br J Surg. 2019 Mar;106(4):404-11.
39. Sadowski SM, Soardo P, Leuchter I, Robert JH,Triponez F. Systematic use of recurrent laryngeal nerve neuromonitoring changes the operative strategy in planned bilateral thyroidectomy. Thyroid. 2013 Mar;23(3):329-33.

40. Schneider R, Randolph GW, Dionigi G,Wu CW, Barczynski M, Chiang FY, et al. International neural monitoring study group guideline 2018 part I: staging bilateral thyroid surgery with monitoring loss of signal. Laryngoscope. 2018 Oct;128 Suppl 3:S1-17.

41. Genther DJ, Kandil EH, Noureldine SI, Tufano RP. Correlation of final evoked potential amplitudes on intraoperative electromyography of the recurrent laryngeal nerve with immediate postoperative vocal fold function after thyroid and parathyroid surgery. JAMA Otolaryngol Head Neck Surg. 2014 Feb;140(2):124-8.

42. Barczynski M, Randolph GW, Cernea C; International Neural Monitoring Study Group in Thyroid and Parathyroid Surgery. International survey on the identification and neural monitoring of the EBSLN during thyroidectomy. Laryngoscope. 2016 Jan;126(1):285-91.

43. Caragacianu D, Kamani D, Randolph GW. Intraoperative monitoring: normative range associated with normal postoperative glottic function. Laryngoscope. 2013 Dec;123(12):3026-31.

44. PavierY, Saroul N, Pereira B, Tauveron I, Gilain L, Mom T. Acute prediction of laryngeal outcome during thyroid surgery by electromyographic laryngeal monitoring. Head Neck. 2015 Jun;37(6):835-9. 